\title{
Beliefs in Misinformation and Conspiracy Theories About COVID- 19: a Triangular Relationship Between Analytic Thinking, Depressive States, and Misperception
}

\section{Marco Delmastro ( $\square$ m.delmastro@agcom.it )}

Autorità per le Garanzie nelle Comunicazioni (AGCOM)

Marinella Paciello

Uninettuno Telematic International University

\section{Research Article}

Keywords: Misinformation and Conspiracy Theories, COVID-19, triangular relationship, analytic thinking, depressive states, misperception

Posted Date: January 4th, 2022

DOI: https://doi.org/10.21203/rs.3.rs-1131403/v1

License: (c) (1) This work is licensed under a Creative Commons Attribution 4.0 International License.

Read Full License 


\section{Abstract}

Beliefs about misinformation and conspiracy theories are often associated with a state of mind. With the spread of the pandemic there has been an outbreak of depression cases among the population. In this paper, we attempt to test the relationship between affective states and beliefs in misinformation about COVID-19 during the early phase of the pandemic. We do this through a survey carried out on a random and representative sample of the Italian population that allows us to go and verify the co-evolution of many factors: i.e., beliefs in misinformation, symptoms of depression, perceptions and misperceptions about COVID-19, ways in which citizens got informed about the pandemic, and sociodemographic characteristics (e.g., age, gender, education). The results show that the relationship between affective state and beliefs in misinformation exists but is more complex than hypothesized.

\section{Introduction}

In 1964, in a seminal paper, Richard Hofstadter defined belief in conspiracy theories (herein also CTs) as "a style of mind", a "paranoid style". In essence, his view was that this style of mind was "a persistent psychic phenomenon, more or less constantly affecting a modest minority of the population". This pioneering analysis has led to a double positive effect of making science interested in the subject and studying it from a psychological perspective (not only political and/or historical).

However, as argued by Brotherton (2015), this approach also influenced the subsequent debate by relegating those who believe in conspiracy theories and, more generally, misinformation to a small group of paranoids. Recent surveys (see, among others, Ibbetson, 2021) show on the contrary that believing in conspiracy theories and misinformation is widespread among the world population and covers the most disparate issues from vaccines to aliens, from politics to climate change.

On the other hand, the persistence in time of the phenomenon, confirmed by historical anecdotes (Brotherton, 2015) and some longitudinal studies (Uscinski \& Parent, 2014), highlights that beliefs in false narratives are a structural phenomenon, typical of all popular cultures.

In this new vein, beliefs in false narratives have been correlated with individuals' negative emotional states. In psychological literature (Freeman, 2007), several studies have already begun to assess the effect exerted by negative emotions exacerbating paranoid thinking, in both clinical and non-clinical populations (Freeman et al. 2011). The negative emotional states such as depressive ones increase the self-perception of vulnerability making people more distressed, especially under stressful conditions (e.g., on-going stress, major life events). The self-perception of being low-power individuals can lead to believe that one's life is controlled by external forces and other persons (Mirowsky \& Ross, 1983) and to react in hostile and aggressive ways (Schaerer et al., 2021). This kind of functioning characterizes particularly paranoid delusion reflecting an insecurely constructed self (Trower and Chadwick,1995). This 'poor me' paranoia is associated not only with depressive feelings but also specific information-processing biases such as "jump to conclusions" in the face of probabilistic information and misunderstanding the 
intentions of others (Freeman \& Garety, 2014). In accordance with this perspective, Abalakina-Paap et al. (1999), in a seminal paper, showed that "beliefs in conspiracies are related to feelings of alienation, powerlessness, hostility, and being disadvantaged. There was no support for the idea that people believe in conspiracies because they provide simplified explanations of complex events". More recently, Swami et al. (2016) confirm that stress (but not anxiety) may be antecedents of beliefs in conspiracy theories. Moreover, other studies show that a stronger belief in conspiracy theories is significantly associated with lower analytic thinking and greater intuitive thinking (Swami et al., 2014).

Coming to the present day, the outbreak of the COVID-19 pandemic has constituted a major threat to physical and mental health (Smith et al., 2021; O'Connor et al., 2021). It has exacerbated depressive symptoms (see among others Rajkumar, 2020) and has questioned one's own personal control over life outcomes. In addition, the pandemic has exacerbated the effect of situations of physical isolation (partly because of the adopted distancing and lockdown restrictions taken by governments to contrast the pandemic) that are likely to alter the mental states (Delmastro \& Zamariola, 2020) and beliefs of citizens. These emotional, cognitive and social elements can facilitate the spread of conspiracy beliefs and more generally misinformation narratives during persistent stressful COVID-19 conditions (De Coninck et al., 2021; Khun et a. 2021).

While many fake news may be harmless and may even be entertaining, the ones related to medical and public health topics can be particularly dangerous for the individual and collective well-being. This latter category "includes misinformation and conspiracy theories related to COVID-19, which is likely one of the most significant pandemics of our lifetime" (Stein et al., 2021).

The relationship between mental states and beliefs in false narratives may be mediated by misperceptions about COVID-19 (Pennycook et al., 2020). Indeed, states of depression and sense of vulnerability can lead citizens not to fully perceive a new and complex phenomenon whose knowledge has changed drastically and quickly over time. Misinformation thus finds a fertile environment where COVID-19 is not properly understood by many citizens within a cognitive framework based on trusted scientific findings.

In this context, much depends on how citizens acquire information on COVID-19 (De Coninck et al., 2021). Previous findings show that conspiracy thinking may be associated with avoidance of mainstream news media and with a tendency to acquire news and information mainly through alternative news sources, such as those shared via social media (Cinelli et al., 2020). In addition, algorithms in social media platforms can lead to echo chambers and confirmation bias among users, which is the main issue in believing in misinformation and fake news (Brugnoli et al., 2019, Cinelli et al., 2021). On the other hand, scientific and institutional sources have become established among part of the population at this stage and many citizens have started to consult directly to acquire accredited and validated news and information on the epidemic and its development

Given the complexity of the subject, the article aims to test some of the previous research hypotheses using a multidisciplinary approach (Delmastro, 2021, van Bavel et al., 2020). 
First, it is important to check whether the worsening of the depressive symptoms due to the pandemic is also correlated with greater gullibility of individuals towards misinformation narratives. In this regard, it is crucial not only to assess the existence of a link between the two phenomena, but also to assess its eventual dimension.

This relationship, whatever it is, clearly coevolves with other factors that must be considered in the analysis. Indeed, the direct (i.e., personal), indirect (i.e., in the family), and contextual (i.e., in the geographic area where individuals live) health effects of COVID-19 on individuals should be considered. As for any social phenomenon, beliefs may depend, even in a completely unexpected way (see the case of vaccines), on the distance from the phenomenon. It is therefore important to verify the co-evolution of beliefs with the spread of the pandemic according to the "distance" of the latter from the individual.

In this context, it is useful to check whether more analytical thinking can help to disengage false beliefs, which as mentioned in the case of health phenomena can have very undesirable effects on public health and social welfare. This is especially true in the case of a pandemic in which the coevolution of the spread of COVID-19 with mental illness, may trigger a syndemic process (Horton, 2020, Singer et al. 2017). To do so, educational attainment may be used as a proxy for individuals' analytical tools. This has two undoubted benefits (and of course some drawbacks, for a discussion see Deary \& Johnson, 2010). First, educational attainment offers an objective element that, although it varies across geographic contexts (but this issue is less prominent in the case of an analysis conducted in a single nation), is less volatile than ad-hoc tests. In addition, the educational system, including permanent education, is a "variable" on which governments can and should operate.

The relationship between the depressive states, analytical tools and beliefs can be mediated by the (mis)perception of phenomena. It is therefore interesting to verify to what extent beliefs in misinformation and conspiracy theories (about COVID-19) are associated with a misperception of the phenomenon and/or by a more adherent (or on the contrary skeptical) attitude towards them.

To do so, we conducted a study on a random and representative sample of the Italian population (aged 16+) right after the first lockdown phase (i.e., in June 2020), to collect the immediate reactions of citizens to the emergency. Indeed, we carried out the survey as soon as the lockdown phase was just over (i.e., socalled "Phase 1"), with the re-opening of manufacturing industries and construction sites and the restart of movements across Italian regions (from 3rd of June). This timeframe appears ideal to conduct a field analysis because the whole lockdown phase had just ended, the citizens' memory of the period just passed was still intact and the organization of such a complex survey was feasible.

\section{Method}

\section{Design and procedure}

We collected data through an online survey among a sample of the Italian population aged $16+$. The design comprehends a wide range of aspects, from feelings and mood, to the perception of COVID-19 
and assessment over several news, real and false, that have been circulated online on the epidemic. Sociodemographic characteristics were also assessed. The survey contained closed-ended questions only and lasted an average of 20 min per participant.

The survey was fielded from June 4, 2020 to June 19, 2020.

In this context, the interviewees were informed that all their personal data would be acquired anonymously and in full compliance with privacy laws.

All methods were carried out in accordance with the Declaration of Helsinki. The experiment was approved by the Ethics Committee of Autorità per le Garanzie nelle Comunicazioni. Informed consent was obtained from all participants and from a parent and/or legal guardian for participants under 18.

Data analysis was performed using Stata Statistical Software and R.

\section{Sample characteristics}

Recruitment and data collection were carried out by a polling firm, to ensure the construction of a random and representative sample of the Italian population (16+). Participants were randomly recruited from census panels. Significant efforts were made to maximize the representativeness of the sample by using software generating representative samples of the population and by including hard-to-reach groups through targeted recruitment.

The final sample was composed of 4,972 Italian individuals, representative of the Italian population in terms of age, gender, and geographical area. More specifically, the sample design and the stratification were based on the following variables: $i)$ age (7 age groups: $16-17$; 18-24; $25-34 ; 35-44$; 45-54; 55-64: 65+); ii) gender, iii) geographical breakdown (all Italian regions and size of the residential community; 7 classes), iv) education (2 classes: graduates and non-graduates).

All responses were anonymized to fully respect the privacy of participants.

\section{Measures}

The measures introduced in the analysis concern the following aspects: beliefs of the participants in misinformation on COVID-19 (and CTs); type of sources used by participants to get informed about the pandemic; psychological condition of participants related in particular to anxiety and depression; sociodemographic characteristics (including territorial control variables relating to the area in which the participants live). In addition, given the nature of the phenomenon under investigation, information has been acquired about the either direct (i.e., personal) or indirect (in the family) experience of participants of COVID-19 disease.

To measure these aspects, a multidisciplinary research approach has been adopted, including tools raging from psychology (for the assessment of mood and feelings), sociology (for the news sources and 
socio-demographic characteristics), and behavioural science (for beliefs in misinformation and perceptions about COVID-19).

\section{Beliefs in misinformation (and conspiracy theories) about COVID-19}

A direct approach has been chosen to assess the gullibility of individuals with misinformation and conspiracy theories (Allcott \& Gentzkow, 2017). The participants were presented with 6 news about the COVID-19 spread on the web, of which 3 were real and 3 false $^{1}$. The news was considered false based on an assessment by third-party independent fact-checkers (i.e., Facta, Newsguard and Pagella Politica) ${ }^{2}$. News was randomly extracted from 12 news reports, without the participants knowing if and how many were real or false. The participants had to assess for each of the news if it was either false or real. Consequently, we can measure beliefs in misinformation by directly assessing, for any false news, whether the individual has correctly assessed its degree of reliability.

\section{COVID-19 information sources}

Participants were asked about the sources of information they used to get informed about COVID-19. The questions concerned all major sources of information (traditional, algorithmic and non-traditional), including institutional sources (such as the Ministry of Health, the Department of Civil Protection, and the World Health Organisation) that in the pandemic period have seen an exponential growth of direct contacts by citizens.

\section{Health: COVID-19 and depressive states}

For this research, a first health factor concerns direct experience with COVID-19. This aspect is essential in understanding the cognitive dynamics of citizens compared to the phenomenon under investigation (i.e., beliefs in misinformation about COVID-19). So, participants were asked whether from the beginning of the pandemic (at the time of the survey 4 months had passed since the outbreak of the virus in Italy ${ }^{3}$ ) they or their family members had been negatively affected by COVID-19.

To evaluate mood and feelings of individuals a psychometric approach was adopted. Due to the special nature of the period which prevented conducting experimental studies meeting participants face-to-face, a psychometric self-reporting methodology was chosen. We adopted the short version of the Mood and Feelings Questionnaire (SMFQ; Messer et al., 1995) which includes 13 items indicating how much individuals have felt or acted depressed during the last few weeks (e.g., "I felt miserable or unhappy", "I felt lonely"). The answers are given on a three-point scale where respondents are asked to decide if the statements are "true", "sometimes true", or "not true". Scoring of the SMFQ is obtained by summing together the point values of responses for each item. The response choices and their designated point values are as follows: "not true" = 0 points, "sometimes true" $=1$ point, "true" $=2$ points. Higher scores on the SMFQ indicate more severe anxiety and depressive symptoms. The range of scores on the SMFQ varies from 0 to 26 . A score of 12 or higher may indicate the presence of anxiety and depression in the 
respondent (see among others Thabrew et al., 2018). For the full discussion on this measure and its implications, see Delmastro and Zamariola (2020).

\section{Socio-demographic characteristics}

Respondents were asked to indicate age (in terms of the number of years since birth). Gender was measured by three options ( $1=$ female, 2 = male, 3 = other), where "female" was used as the control category in the estimates (see below). Information regarding educational attainment (i.e., the highest level of education that a person has successfully completed) was codified as follows: $0=$ no degree; 1 : elementary education, $2=$ middle school; 3 = high school; 4 = graduation; $5=$ master degree/Ph.D. A dummy variable has also been introduced which takes the value of 1 when the individual lives alone (it is 0 otherwise). Finally, geographical control variables (see Table A.2 in the Appendix) have been introduced that measure the size of the municipality in which the individual lives and the spread of COVID-19 in the same area at the time of the survey (31 May 2020).

\section{Perception of COVID-19}

A final aspect analyzed in the paper concerns (mis)perceptions of the participants about COVID-19 (Pennycook et al., 2020). To do this, the participants were given 5 multiple choice questions regarding the main features of COVID-19 known at the time of the survey. Accordingly, an indicator of bias in perception has been constructed that takes value 1 (maximum) in the case of all wrong answers, is equal to 0 (minimum) in the opposite case in which participants have successfully answered all questions and is between 0 and 1 in all other cases.

${ }^{1}$ Some of the fake news belongs to conspiracy theories circulating about the origin of the coronavirus (see below).

${ }^{2}$ It is important to note that in the econometric models have been also included control variables related to each news: i.e., the type of news (i.e., COVID-19 prevention, treating, and current affairs) and its diffusion on social networks (i.e., the number of actions).

${ }^{3}$ The virus was first confirmed to have spread to Italy on January 31,2020 , when two Chinese tourists in Rome tested positive for the virus.

\section{Results}

As a first step, we analyze the correlation between beliefs in misinformation and three sets of factors: the socio-demographic characteristics of individuals, how they got informed (about COVID-19), and the state of health (see Table A.1 in the Appendix).

From a statistical point of view, an econometric analysis was performed that allows all factors to be considered together (including geographic control variables and those related to individual news items i.e., type and diffusion). Since each participant was administered 6 news items on COVID-19, 3 of which 
were false (see method section), the model used is a probit (in which the dependent is 1 if the individual believes a false news item and 0 otherwise) panel date (with the number of observations equal to the responding individuals times 3 , i.e., the number of false news items submitted to each participant). Results are presented in Table 1 (model I).

Interestingly, all else being equal, people who live alone are significantly (at 95\%) more likely to believe fake news. This relationship shows how false beliefs are most associated with situations of physical isolation, which became dramatically more substantial during the pandemic (with the measures taken by governments to contrast the spread of the virus that led Italy to lockdown restrictions during the first phase of the outbreak, see above).

Conversely, educational attainment emerges as a decisive factor ( $99 \%$ significant) in explaining nonbeliefs in misinformation. The development of analytical tools due to a more solid educational background appears to be a strong deterrent to gullibility concerning misinformation and conspiracy narratives.

How individuals get informed is highly correlated to their likelihood to believe in misinformation. First, the more individuals consult different sources, the less likely they are to believe fake news. This indicates how much access to a diversity of viewpoints can develop a critical attitude in the individual. Second, the specific type of (primary) source of news and information about COVID-19 significantly affects individuals' beliefs: people who use alternative, non-accredited sources tend to adhere more readily to narratives of misinformation. An interesting aspect is the widespread direct access of citizens to institutional sources providing information and data about COVID-19 (as many as 35.9\% of the Italian population used institutional sources as their first source of information, see Table 1). This active attitude, not mediated by journalistic activity, appears to significantly reduce individuals' tendency to believe in false narratives (the coefficient is negative and significant at $99 \%$ ).

Turning to emotional states issues, people who developed symptoms of depression appear, with a significantly higher probability, to believe in misinformation. Likely, these negative states could increase beliefs in misinformation because they could confirm the depressive-related ideas that events are uncontrollable, and individuals are vulnerable and powerless.

Turning to the health aspects, it may seem counterintuitive to the result of the COVID-19 variable whose coefficient is positive and significant (at $95 \%)^{4}$. One might have expected that those who have already experienced the disease (themselves or their families) have more information available to them and thus are less likely to believe in false narratives ${ }^{5}$. Alternatively, these negative experiences, which are associated with depressive feelings, could increase beliefs in misinformation because they confirm the perception of powerlessness in dealing with so harmful events. However, given that available data (i.e., cross-section) the estimates do not allow to identify causal relationships, so that the opposite relationship may have prevailed: i.e., individuals who believe in misinformation narratives are less able to manage Covid-related events and expose themselves more to the dangers of disease contagion ${ }^{6}$. 
We have then proceeded to estimate, based on a linear panel data model, the value of the probability of believing in misinformation about COVID- $19^{7}$ for different values of the significant explanatory variables (Table 2$)^{8}$. The probability decreases from $45-33 \%$ going up the level of educational attainment. In contrast, the probability increases from $38-45 \%$ for increasing levels of depression. The size of the variation in the case of different media mixes is also significant, in terms of both the number of news sources used to get informed about COVID-19 (from $41-33 \%$ for an increasing number of sources) and the type of primary news sources ( $47 \%$ in the case of non-traditional sources and $34 \%$ in the case of institutional sources).

Since the factors considered may vary jointly (e.g., those who live alone are more likely to be depressed, see Delmastro \& Zamariola, 2020) we also compared three possible and opposite types of individuals: type A (i.e., people who are well-educated and well-informed, live in a family, and have a good mood), type $B$ (i.e., people who are less educated, depend only on few non-established sources of news about COVID19, live alone, and exhibit severe symptoms of depression), and the benchmark case (Table 3). The probability of believing in misinformation increases from a value of one-quarter (type $A$ ) to nearly threequarters (type B).

\section{Belief in CT and mood}

If we analyze only a fake news that contains a conspiracy theory about COVID-19 $19^{9}$ then the effect of mood and feelings on beliefs is even more evident: those who have a normal mood (SFMQ index equal to 11 or less) believe only $13 \%$ of the time in a conspiracy theory, while this percentage rises to $33 \%$ for individuals with symptoms of depression (SFMQ index equal to 12 or more). This result is in line with the 'poor me' paranoid idea associated with depressive feelings and with also a misunderstanding of other intentions (Freeman \& Garety, 2014).

Figure 1 shows the distribution of the SFMQ index for two categories of individuals: those who believe and those who do not believe in a conspiracy theory about COVID-19. From the boxplot it is evident that those who believe in a conspiracy theory display a significantly higher level of depression.

\section{Bias in perception and belief in misinformation}

The next step is to analyze whether (and how much) beliefs in misinformation narratives are related to misperception, i.e., a bias in the perception of COVID-19.

To do this, as explained above, participants were asked 5 multiple choice questions about the hitherto known characteristics of COVID-19. Based on the answers of participants, an index of the bias in perception of the phenomenon has been constructed, that is equal to 0 when the individual has guessed all the answers (maximum), is 1 when instead it has mistaken all the answers (minimum) and is between 0 and 1 in all other cases. We have included this variable in the previous probit panel data model. The results are shown in Table 1 (model II). 
This variable, as expected, is very significant (at 99\%) and positive, highlighting a strong correlation between misperception bias and beliefs in misinformation. If we calculate the probability of believing in false narratives about COVID-19 depending on the bias in perception (Figure A.1), we observe that starting from a minimum level around $30 \%$ (of the benchmark case) the probability grows to over $60 \%$ in the case of maximum misperception, essentially doubling its value.

But the most interesting results are on the one hand the loss of explanatory value of the mood variable, and on the other the confirmation instead of the significance of educational attainment. In other words, mental state appears to affect the beliefs of individuals only through a bias in perception of COVID-19.

\section{Analytic thinking, mental states, bias in perception, and beliefs in misinformation}

If we go deep into the relationship between (mis)perception, educational attainment and mental states, interesting results come out.

First, we can study the distribution of the perception bias for categories of individuals with different educational attainment (Figure A.2) and mental state (Figure A.3). A clear positive relationship emerges between depression (SMFQ index equal to 12 or more) and bias in perception. There is also a negative relationship between perception bias and educational attainment, in which it is the lack of minimum analytic tools that makes the difference. In others, the effect of education is significant until middle school, after which the level of bias in perception is substantially stable.

Second, if we estimate an econometric model (a double-censoring count model, see Table A.1 in Appendix) it emerges that such relationships (i.e., between misperception and educational attainment and mental states) are significant even when we consider all factors taken together.

In conclusion, as depicted in Figure 2, we can conclude that while the educational attainment appears to directly (and negatively) impact both misperceptions and beliefs in false narratives, the effect of mental state appears to be only indirect, i.e., mediated by a bias in perception.

${ }^{4}$ It should be noted that beliefs in fake news are instead negatively (and significantly) correlated with the spread of contagion in the area where citizens live (i.e., geographical control variables).

${ }^{5}$ As noted in the previous note, this applies in the case of contagions in the same area where the individual lives.

${ }^{6}$ The relationship between misinformation beliefs and number of confirmed cases in the area where citizens live is different because the latter represents an exogenous component for individuals. In this case, as mentioned, the relationship is significant and negative.

${ }^{7}$ Although the experimental nature of the exercise requires that the estimates be taken with caution, given that the sample is random and representative of the population, these can be considered as estimates of 
the entire Italian population (16+).

${ }^{8}$ These simulations, based on an econometric model that takes all factors into account simultaneously, avoid (or at least significantly reduce) spurious correlation phenomena.

${ }^{9}$ It was submitted to sample individuals a news circulated online according to which "The coronavirus strain that is spreading in China and abroad is a patented virus owned by an entity called The Pirbright Institute, partially funded by the Bill and Melinda Gates Foundation". This news appears to fully satisfy the definition of conspiracy theory according to which "'Conspiracy theories" are attempts to explain the ultimate causes of significant social and political events and circumstances with claims of secret plots by two or more powerful actors" (Douglas, 2019; see also Brotherton, 2015).

\section{Discussion}

Beliefs in misinformation (going as far as conspiracy theories) affect a large portion of the population and may be due to various reasons (Pennycook \& Rand, 2021). The article explored some factors that predispose individuals to be naturally more responsive and less skeptical about such false narratives. When regarding information about COVID-19 such beliefs assume a crucial importance because they may induce behaviors dangerous for the individual and collective well-being.

A relevant aspect is the relationship between mental states and beliefs in misinformation. In this research, it was shown that people who suffer from symptoms of depression are more likely to believe in misinformation about COVID-19. In the past few months, the psychological literature has shown how the pandemic has caused parts of the population to develop these symptoms (Rajkumar, 2020). By connecting these research results, it is evident how there is a concrete risk of creating a social process characterized by negative feedback loops: from the pandemic to depressive symptoms, that lead to false beliefs about COVID-19, which in turn can generate socially inappropriate behaviors that fuel the spread of the pandemic, and so on.

As noted in the article, this process is mediated by misperception. Depression can alter the perception of the phenomenon inducing individuals to believe more in fake news and conspiracy theories about COVID19. In line with literature (Freeman, 2007; Freeman et al. 2011; Schaerer et al. 2021), this result underline the strong interplay among depressive states and paranoid thinking during Covid-19. Misinformation can root in a dysfunctional affective-cognitive state that could become chronically activated and maintained by it. This result sheds the light on the importance of psychologically supporting those individuals that during long period of stress and isolation might be more at risk to develop resigned attitude toward themselves and life events.

It has also been noted that individuals' analytical tools act directly on false beliefs (as well as indirectly on misperception), triggering an attitude of natural skepticism. Phenomena of functional illiteracy that are too often neglected in our society are fuel for false beliefs, especially as said in such stressful times for individuals.

Page $11 / 18$ 
Moreover, the central role of misperception poses a problem of the effectiveness of scientific communication (especially about COVID-19). Research findings show how institutional sources can represent a significant barrier to the spread of misinformation. However, this is not enough because there is a large part of the population that gets informed only by a few, unaccredited sources of news and that, in any case, also for psychological reasons, is rather immune to information coming from traditional media and institutions.

\section{References}

1. Abalakina-Paap M, Stephan WG., Craig T, Gregory WL. Beliefs in Conspiracies, Political Psychology, 1999: 20(3), 637-647.

2. Allcott H, Gentzkow M. Social media and fake news in the 2016 election. Journal of Economic Perspectives, 2017: 31(2), 211-236.

3. Brotherton R. Suspicious Minds. Why We Believe Conspiracy Theories, Bloomsbury Sigma, 2015.

4. Brugnoli E, Cinelli M, Quattrociocchi W, Scala A. Recursive patterns in online echo chambers. Scientific Reports, 2019: 9(20118).

5. Cinelli M, Quattrociocchi W, Galeazzi A, Valensise CM, Brugnoli E, Schmidt AL, Zola P, Zollo F, Scala A. The COVID-19 social media infodemic. Scientific Reports, 2020: 10(16598).

6. Cinelli M, De Francisci Morales G, Galeazzi A, Quattrociocchi W, Starnini M. The echo chamber effect on social media, Proceedings of the National Academy of Sciences, 2021: 118(9).

7. Deary IJ, Johnson W. Intelligence and education: causal perceptions drive analytic processes and therefore conclusions. International journal of epidemiology, 2010: 39(5), 1362-1369.

8. De Coninck D, Frissen T, Matthijs K, d'Haenens L, Lits G, Champagne-Poirier O, Carignan ME, David MD, Pignard-Cheynel N, Salerno S, Généreux M. Beliefs in Conspiracy Theories and Misinformation About COVID-19: Comparative Perspectives on the Role of Anxiety, Depression and Exposure to and Trust in Information Sources. Front Psychol. 2021 Apr 16;12:646394.

9. Delmastro M. On the Measurement of Social Phenomena: A Methodological Approach. Springer Nature, 2021.

10. Delmastro M, Zamariola G. Depressive symptoms in response to COVID-19 and lockdown: a crosssectional study on the Italian population. Scientific reports, 2020: 10(1), 1-10.

11. Douglas KM, Uscinski JE, Sutton RM, Cichocka A, Nefes T, Ang CS, Deravi F. Understanding conspiracy theories. Political Psychology, 2019: 40, 3-35.

12. Freeman, D. Suspicious minds: the psychology of persecutory delusions. Clinical psychology review, 2007: 27(4), 425-457.

13. Freeman, D., \& Garety, P. Advances in understanding and treating persecutory delusions: a review. Social Psychiatry and Psychiatric Epidemiology, 2014: 49(8), 1179-1189.

14. Freeman, D., McManus, S., Brugha, T., Meltzer, H., Jenkins, R., \& Bebbington, P. Concomitants of paranoia in the general population. Psychological Medicine, 2011: 41(5), 923-936. 
15. Hofstadter R. The Paranoid Style in American Politics, Harper's Magazine, 1964: 1374, 77-86.

16. Horton R. Offline: COVID-19 is not a pandemic, Lancet, 2020:396, 874.

17. Ibbetson C. Where do people believe in conspiracy theories? YouGov, 2021: https://yougov.co.uk/topics/international/articles-reports/2021/01/18/global-where-believeconspiracy-theories-true.

18. Jarbin H, Ivarsson T, Andersson M, Bergman H, Skarphedinsson G. Screening efficiency of the mood and feelings questionnaire (MFQ) and short mood and feelings questionnaire (SMFQ) in Swedish help seeking outpatients. 2020: PLoS One 15.

19. Kuhn, S. A. K., Lieb, R., Freeman, D., Andreou, C., \& Zander-Schellenberg, T. Coronavirus conspiracy beliefs in the German-speaking general population: endorsement rates and links to reasoning biases and paranoia. Psychological Medicine, 2021: 1-15.

20. Messer S, Angold A, Costello J, Van Kämmen W, Stouthamer-Loeber M. Development of a short questionnaire for use in epidemiological studies of depression in children and adolescents: factor composition and structure across development. Int. J. Methods Psychiatr. Res., 5, 1996: 251-262.

21. Mirowsky, J., \& Ross, C. E. (1983). Paranoia and the structure of powerlessness. American Sociological Review, 228-239.

22. O'Connor, R. C., Wetherall, K., Cleare, S., McClelland, H., Melson, A. J., Niedzwiedz, C. L., ... \& Robb, K. A.. Mental health and well-being during the COVID-19 pandemic: longitudinal analyses of adults in the UK COVID-19 Mental Health \& Wellbeing study. The British Journal of Psychiatry, 2021: 218(6), 326-333.

23. Pennycook G, McPhetres J, Bago B, Rand, D. G. Predictors of attitudes and misperceptions about COVID-19 in Canada, the UK, and the USA. PsyArXiv, 2020: 10, 1-25.

24. Pennycook G, Rand DG, The psychology of fake news. Trends in cognitive sciences, 2021.

25. Rajkumar RP. COVID-19 and mental health: A review of the existing literature. Asian J. Psychiatr. 2020: 52, 102066.

26. Schaerer, M., Foulk, T., du Plessis, C., Tu, M. H., \& Krishnan, S. Just because you're powerless doesn't mean they aren't out to get you: Low power, paranoia, and aggression. Organizational Behavior and Human Decision Processes, 2021: 165, 1-20.

27. Singer M, Bulled N, Ostrach B, Mendenhall E. Syndemics and the biosocial conception of health, Lancet, 2017: 389, 941-950.

28. Smith, A. M., Willroth, E. C., Gatchpazian, A., Shallcross, A. J., Feinberg, M., \& Ford, B. Q. Coping with health threats: The costs and benefits of managing emotions. Psychological Science, 2021: 32(7), 1011-1023.

29. Swami V, Voracek M, Stieger S, Tran US, Furnham A. Analytic thinking reduces belief in conspiracy theories. Cognition, 2014: 133(3), 572-585.

30. Swami, V, Furnham, A, Smyth, N, Weis, L, Lay, A, Clow A. Putting the stress on conspiracy theories: Examining associations between psychological stress, anxiety, and belief in conspiracy theories. 
Personality and Individual Differences, 2016: 99, 72-76.

31. Stein R A, Ometa O, Shetty SP, Katz A, Popitiu MI, Brotherton R. Conspiracy theories in the era of COVID-19. A tale of two pandemics. International Journal of Clinical Practice, 2021: 75(2).

32. Thabrew H, Stasiak K, Bavin LM, Frampton C, Merry S. Validation of the Mood and Feelings Questionnaire (MFQ) and Short Mood and Feelings Questionnaire (SMFQ) in New Zealand helpseeking adolescents. Int. J. Methods Psychiatr. Res.,2018: 27, 1-9.

33. Trower, P., \& Chadwick, P. Pathways to defense of the self: A theory of two types of paranoia. Clinical Psychology: Science and Practice, 1995: 2(3), 263.

34. Turner N, Joinson C, Peters TJ, Wiles N, Lewis G. Validity of the Short Mood and Feelings questionnaire in late adolescence. Psychol. Assess., 2014: 26, 752-762.

35. Uscinski, JE, Parent, JM. American conspiracy theories. Oxford University Press, 2014.

36. Van Bavel, et al. Using social and behavioural science to support COVID-19 pandemic response. Nature Human Behaviour, 2020: 30, 1-12.

\section{Tables}

Table 1: Result of probit panel data models 


\begin{tabular}{|c|c|c|c|}
\hline \multirow[b]{2}{*}{ Categories } & \multirow[b]{2}{*}{ Variables } & (1) & (2) \\
\hline & & \multicolumn{2}{|c|}{ Belief in fake news } \\
\hline \multirow{2}{*}{ Socio-demo } & Gender (female $=1$ ) & $0.0551^{*}$ & $0.0748^{* *}$ \\
\hline & & $(0.0311)$ & $(0.0306)$ \\
\hline \multirow[t]{2}{*}{ Socio-demo } & Age (number of years) & 0.000178 & -0.000957 \\
\hline & & $(0.000979)$ & $(0.000963)$ \\
\hline \multirow[t]{2}{*}{ Socio-demo } & Living alone $(=1)$ & $0.0935^{* *}$ & $0.106^{* *}$ \\
\hline & & $(0.0450)$ & $(0.0444)$ \\
\hline \multirow[t]{2}{*}{ Socio-demo } & Education (6 degrees, increasing order) & $-0.0712^{* * *}$ & $-0.0586^{* * *}$ \\
\hline & & $(0.0196)$ & $(0.0192)$ \\
\hline \multirow[t]{2}{*}{ News about COVD-19 } & Number of news sources $(0-22)$ & $-0.0105^{* *}$ & -0.00547 \\
\hline & & $(0.00434)$ & $(0.00424)$ \\
\hline \multirow[t]{2}{*}{ News about COVD-19 } & Non-traditional news outlets $(=1)$ & $0.257 * * *$ & $0.137^{* * *}$ \\
\hline & (tra ditional sources = benchmark) & $(0.0526)$ & $(0.0522)$ \\
\hline \multirow[t]{2}{*}{ News about COVID-19 } & Algorithmic news sources $(=1)$ & $0.115^{* *}$ & 0.0137 \\
\hline & (traditional sources = benchmark) & $(0.0582)$ & $(0.0580)$ \\
\hline \multirow[t]{2}{*}{ News about COVID-19 } & Institutional news sources $(=1)$ & $-0.117^{* * *}$ & $-0.0770^{* *}$ \\
\hline & (traditional sources = benchmark) & $(0.0329)$ & $(0.0323)$ \\
\hline \multirow[t]{2}{*}{ Perception about COVD-19 } & Bias in perception & & $0.899 * * *$ \\
\hline & (index: $0-1$ ) & & $(0.0739)$ \\
\hline \multirow[t]{2}{*}{ Health } & Mood (SMQF, 0-26) & $0.00808^{* *}$ & 0.00129 \\
\hline & (>11 clinical depression) & $(0.00323)$ & $(0.00319)$ \\
\hline \multirow[t]{8}{*}{ Health } & COVD- 19 in family $(=1)$ & $0.138^{* *}$ & 0.0655 \\
\hline & & $(0.0609)$ & $(0.0594)$ \\
\hline & Constant & 0.119 & -0.0905 \\
\hline & & $(0.137)$ & $(0.135)$ \\
\hline & Geographic controls & YES & YES \\
\hline & News controls & YES & YES \\
\hline & Observations & 13,572 & 13,572 \\
\hline & Number of individuals & 4,524 & 4,524 \\
\hline
\end{tabular}

${ }^{(*)}$ Note: The estimates in the table refer to probit panel data models, with robust standard errors. The dependent variable is 1 when the individual erroneously believes in fake news. For each variable, the coefficient, the standard error (in parentheses), and the level of significance are reported as follows: ** significant at $99 \%$; ** significant at $95 \%$.

Table 2: Probabilities of believing in misinformation for different categories of citizens 


\begin{tabular}{|l|l|r|}
\hline & & Probability of believing in misinformation \\
\hline & Benchmark case $^{(*)}$ & $38.1 \%$ \\
\hline Socio-demo & Living alone (=1) & $41.1 \%$ \\
\hline Socio-demo & Education min (no degree) & $45.1 \%$ \\
\hline & Education mean (high school) & $38.1 \%$ \\
\hline & Education max (post-grad) & $33.4 \%$ \\
\hline News about COVD-19 & Number of news sources: min (0) & $41.2 \%$ \\
\hline & Number of news sources: mean (9) & $38.1 \%$ \\
\hline & Number of news sources: max (22) & $33.6 \%$ \\
\hline News about COVD-19 & Traditional news sources & $38.1 \%$ \\
\hline & Non-traditional news sources & $46.6 \%$ \\
\hline & Institutional news sources & $34.3 \%$ \\
\hline Health & Good mood (SMFQ =0) & $38.1 \%$ \\
\hline & Mood just above threeshold(SMFQ = 12) & $41.2 \%$ \\
\hline Health & Maximum depression (SMFQ =26) & $44.9 \%$ \\
\hline & Covid-19 (=1) & $42.7 \%$ \\
\hline
\end{tabular}

${ }^{(*)}$ Note: The estimates in the table refer to a linear panel data model, with robust standard errors. The benchmark case has been calculated as follows: average age (i.e., years=49), living in a family, highschool education, average number of news sources on COVID-19 (i.e., 9), SMFQ index =12, no COVID-19 in family.

Table 3: Probabilities of believing in misinformation for types of citizens

\begin{tabular}{|l|l|l|}
\hline Type of individual & Characteristics & Probability of believing in misinformation \\
\hline Type A & $\begin{array}{l}\text { Max education, living in } \\
\text { famlily, good mood, get } \\
\text { informed from many sources }\end{array}$ & $25.1 \%$ \\
\hline Benchmark & $\begin{array}{l}\text { Average education, living in } \\
\text { family, few symptoms of } \\
\text { depression, got informed from } \\
\text { fewsources }\end{array}$ & $38.1 \%$ \\
\hline Type B & $\begin{array}{l}\text { No education, living alone, } \\
\text { symptoms of depression, got } \\
\text { informed from only non } \\
\text { traditionional sources }\end{array}$ & $72.0 \%$ \\
\hline
\end{tabular}

${ }^{(*)}$ Note: The estimates in the table refer to a linear panel data model, with robust standard errors. 
Figures

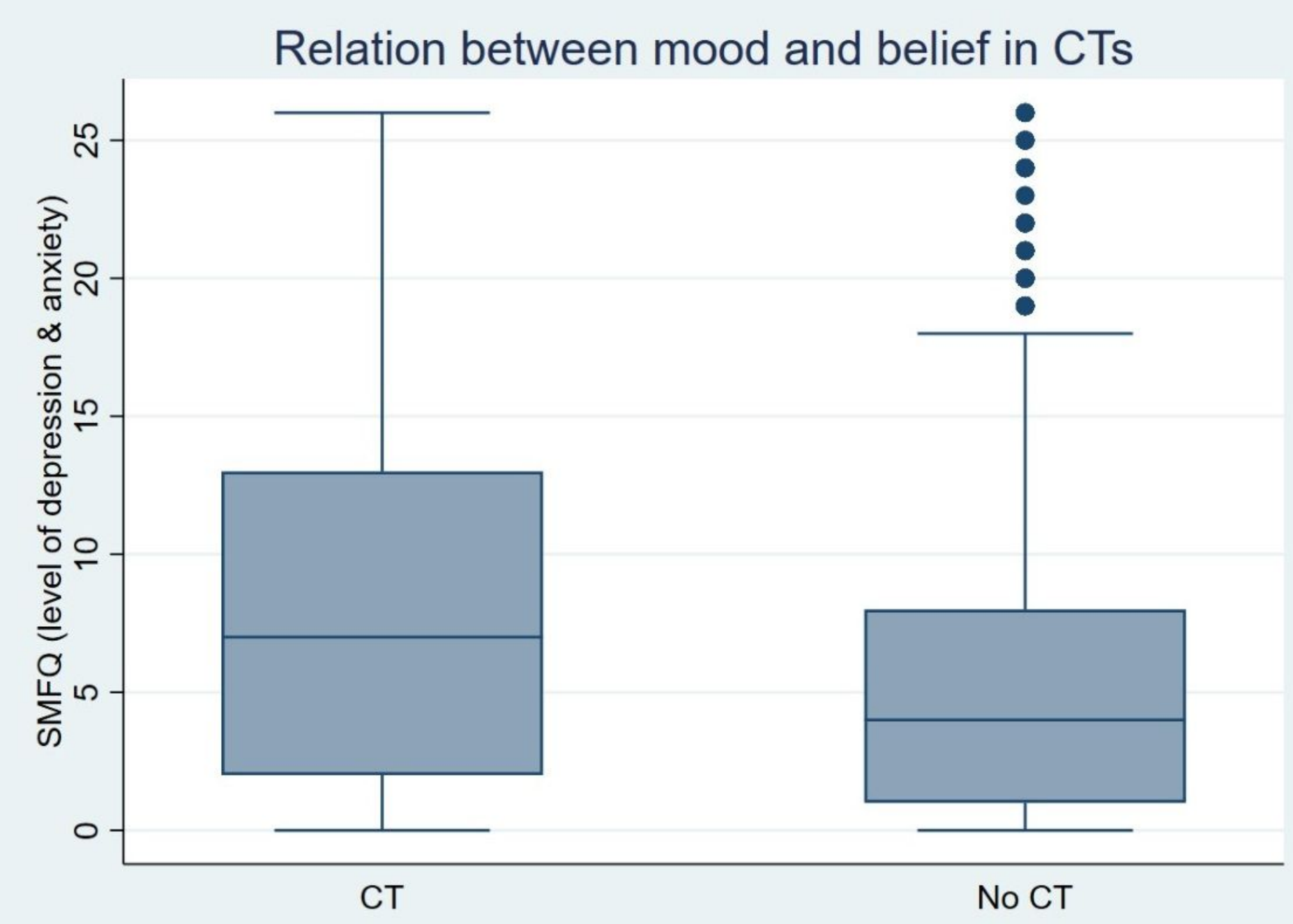

Figure 1

Boxplot of the mood (i.e., SMFQ index) for two categories of individuals: those who believe in a conspiracy theory about COVID-19 (CT) and those who don't (No CT) 


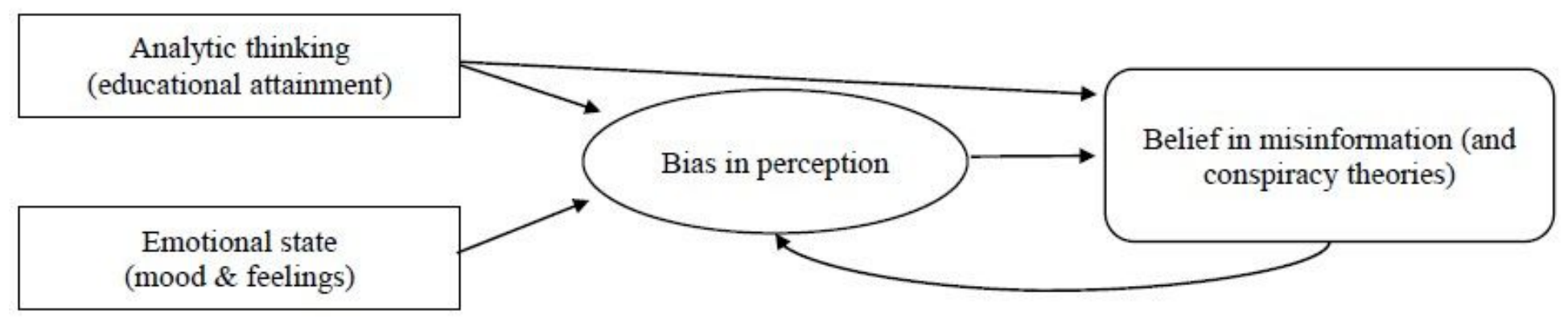

Figure 2

Relations between analytic tools and emotional state, bias in perception, and belief in misinformation

\section{Supplementary Files}

This is a list of supplementary files associated with this preprint. Click to download.

- DisinfoCTMoodDMSRAppendix.pdf 